\title{
THE EFFECTS OF NERVE GROWTH FACTOR AND ITS ANTISERUM ON THE POSTNATAL DEVELOPMENT AND SURVIVAL AFTER INJURY OF SENSORY NEURONS IN RAT DORSAL ROOT GANGLIA ${ }^{1}$
}

\author{
HENRY K. YIP, ${ }^{2}$ KEITH M. RICH, ${ }^{*}$ PATRICIA A. LAMPE, AND EUGENE M. JOHNSON, JR. \\ Departments of Pharmacology and *Neurosurgery, Washington University School of Medicine, St. Louis, Missouri 63110
}

Received January 10, 1984; Revised April 6, 1984; Accepted May 30, 1984

\begin{abstract}
Nerve growth factor (NGF) is known to be essential for survival and maintenance of sympathetic ganglia and of embryonic sensory ganglia of neural crest origin. The present study examined the physiological and pharmacological roles of NGF in the postnatal development of sensory neurons in the dorsal root ganglion (DRG). In contrast to what is generally stated in the literature, administration of NGF antiserum to newborn rats for a period of 7 days resulted in a significant $(\sim 20 \%)$ reduction of neuronal number in the lumbar DRG. Size spectrum analysis of surviving neurons revealed a shift toward larger sizes, presumably due to a preferential loss of small cells.

The number of neurons in the $\mathrm{L}_{5}$ DRG was studied at various times after unilateral sciatic nerve crush in 1day-old rats. Axotomy resulted in a substantial loss (40 to 50\%) of neurons in the immature DRG. Administration of NGF antiserum to animals with axotomized DRG did not increase cell death when compared with the axotomized controls. However, the number of neurons in the antiserum-treated ganglia decreased by the same percentage (20\%) when compared with the control serum-treated ganglia before and after axotomy. Treatment with NGF initially prevented the loss of neurons in the axotomized DRG. However, some neurons died during the first week despite continued NGF administration; and, subsequent to NGF withdrawal, neuronal number decreased to the same level as in control animals. Thus, removal of exogenous NGF resulted in the death of the sensory neurons which had been maintained. These results indicate that cells no longer physiologically dependent on NGF for survival (as indicated by lack of cell death in the presence of NGF antiserum in the axotomized DRG) can be sustained by pharmacological administration of NGF.
\end{abstract}

The cell body response of dorsal root ganglia (DRG) sensory neurons following axonal injury is well documented (Hare and Hinsey, 1940; Pannese, 1963; Carmel and Stein, 1969; Lieberman, 1974). However, the signals which initiate these changes have not been determined. One of the mechanisms postulated is that neurons depend on trophic factors derived from the target cells for their maintenance and survival (Ramón y Cajal, 1928; Hamburger and Levi-Montalcini, 1949; Cragg, 1970; Watson, 1974). 'This interaction between the peripheral target organ and the nerve cell body is particularly important during the early developmental period when the immature neurons are still in active growth and differentiation (Hollyday and Hamburger, 1976; Houthoff and Drukker, 1977; Landmesser and Pilar, 1978; Thoenen et al., 1979).

Nerve growth factor (NGF) is the only well defined agent that plays a role in mediating trophic actions between a neuron and its peripheral target cells (see review by Theonen and Barde, 1980). It is well established that NGF is important for the survival, growth, and differentiation of peripheral sympa-

We thank Dr. Thomas Woolsey for his computer facilities, and Dr. Arthur Loewy and Patricia Osborne for their advice and assistance. This work was supported by National Institutes of Health Grants HL20604 and NS18071.

${ }^{2}$ To whom correspondence should be addressed. thetic and neural crest-derived sensory neurons in vivo and in vitro. Previous studies have shown that NGF is selectively taken up by sympathetic and sensory nerve terminals through a specific receptor-mediated process and is transported retrogradely to the cell bodies where it exerts its trophic effects (Hendry et al., 1974; Paravicini et al., 1975; Stöckel et al., 1975; Johnson et al., 1978).

The most compelling evidence for the biological importance of NGF is that administration of NGF antiserum to immature animals leads to the destruction of their peripheral sympathetic neurons. This degenerative change is the result of the deprivation of endogenous NGF rather than a complement-mediated cytotoxic effect (Ennis et al., 1979; Goedert et al., 1980). Furthermore, these destructive effects of anti-NGF can be almost completely reversed by administration of sufficient doses of NGF within $48 \mathrm{hr}$ after antiserum treatment (Goedert et al., 1980). Administration of NGF to newborn animals during a critical period in postnatal development has been shown to prevent the effects of surgical axotomy and the effects of treatment with 6-hydroxydopamine or drugs that interfere with the axonal transport mechanism (Aloe et al., 1975; Hendry, 1975; Johnson, 1978). These findings are consistent with the proposed role of NGF as a retrograde trophic agent in sympathetic neurons.

In contrast to the continuous dependence of sympathetic neurons on NGF throughout life (Gorin and Johnson, 1980; 
Johnson et al., 1980), DRG sensory neurons have not been shown to require NGF for survival postnatally. Exposure to low amounts of NGF antiserum in vivo does not affect the number of sensory neurons in the cervical DRG in the early postnatal period (Johnson et al., 1980). However, NGF has been demonstrated to be required during embryonic development of sensory neurons (Gorin and Johnson, 1979; Johnson et al., 1980; Hamburger et al., 1981; Aloe et al., 1981). Less is known about any degree of postnatal dependence of DRG neurons on NGF in vivo. Recent studies have indicated that treatment with NGF markedly increases the substance $P$ content of DRG in both newborn and adult rats (Kessler and Black, 1980; Otten et al., 1980; Goedert et al., 1981) and to cause hypertrophy of postnatal DRG neurons (Kornblum and Johnson, 1982). Conversely, exposure of NGF antiserum leads to a significant reduction in substance $P$ levels even in adult animals (Schwartz et al., 1982). These results suggest that, postnatally, sensory neurons in the DRG are indeed responsive to NGF and require NGF for maintenance of at least some functions. Further evidence is that NGF also protects the substance P-containing DRG neurons from the toxic effects of capsaicin in neonatal rats (Otten et al., 1983). All these data indicate that NGF may be an important regulatory agent in the postnatal development of at least some sensory neurons in the DRG.

Marked changes in metabolic activities occur in the cell bodies of neurons after their axons are interrupted (Bratlgard et al., 1957; Watson, 1965; Murray and Grafstein, 1969). The reactive changes which occur following axotomy reflect a significant shift in metabolism, adaptive to injury, often to the level of a more embryonic stage (Bodian, 1947; Brattgard et al., 1957; Watson, 1965). Conceivably, there may be a "regressive" shift of DRG neurons to a prenatal dependence on NGF as a result of axonal injury.

Thus, in the present study, we further define the role of NGF in the survival of the postnatal DRG neurons and determine whether exogenous NGF can decrease the deleterious effects of neuronal injury.

\section{Materials and Methods}

Experimental animals and surgical procedures. Experiments were conducted to determine the rate of neuronal loss in DRG after axotomy in early postnatal development. Newborn Sprague-Dawley rats (Chappel Breeders, St. Louis, MO), on postnatal day (PND)-0, were anesthetized on ice. The right sciatic nerve was exposed and crushed at the tendon of obturator internus. The left sciatic nerve served as an unoperated control. The wound was closed with 6-0 silk suture and the pups were returned to their mother. Success of the surgery was demonstrated by a paralysis of the operated limb.

Preparation of NGF and its antiserum. The 2.5S mouse NGF was purified from male mouse submaxillary glands according to the methods of Bocchini and Angeletti (1969) and stored in lyophilized form until used. For all experiments, an appropriate amount of NGF $(1 \mathrm{mg} / \mathrm{ml})$ was dissolved in $0.9 \%$ phosphate-buffered saline (PBS). This solution was divided into aliquots and kept frozen until needed. Subcutaneous injections either of $100 \mu \mathrm{g}$ of NGF or of an equal volume of vchicle were given to the neonates $2 \mathrm{hr}$ before the operation and continued daily through PND-6. Guinea pig antiserum to mouse NGF was prepared as previously described (Johnson et al., 1980); its titer against mouse NGF was determined by the chick embryonic DRG bioassay (Fenton, 1970; Gorin and Johnson, 1979). Only pooled sera with a high titer of $\mathrm{NGF}$ antibody $(>4000)$ were used in the experiments. Administration of NGF antiserum $(150 \mu \mathrm{l})$ followed the same schedule as the NGF treatment. The superior cervical ganglia (SCG) were examined histologically in order to confirm the effectiveness of NGF and its antisera. Control serum was collected from normal, nonimmunized guinea pigs.

Histological procedures and morphometric analysis. To determine the proportion of $L_{5}$ DRG neurons projecting in the rat sciatic nerve, the right sciatic nerve was crushed in adult rat and $1 \mu \mathrm{l}$ of a $20 \%$ horseradish peroxidase (HRP, Sigma type VI) solution were injected into the lesion site. After $24 \mathrm{hr}$, the animals were anesthetized and perfused through the heart with physiologic saline followed immediately by fixative ( $2.5 \%$ glutaraldehyde, $0.5 \%$ paraformaldehyde in phosphate buffer, $\mathrm{pH} 7.4$ ). The DRG at the level of $\mathrm{L}_{3}$ to $\mathrm{L}_{6}$ were dissected out, postfixed overnight, and stored in a $30 \%$ sucrose buffer solution at $4^{\circ} \mathrm{C}$. The contralateral ganglia served as controls. Longitudinal frozen sections were cut at 40 $\mu \mathrm{m}$. The tissue was processed for HRP using tetramethylbenzidine as a chromagen according to the protocol of Mesulam (1978). Serial sections were mounted on chrom-alum-coated slides and counterstained with thionin. The number of labeled neurons was counted under brightfield illumination; only neurons with a clear cell boundary were counted.

At intervals $(0.5,1,3,4,6,8,10,14$, and 28 days) after the nerve crush, the animals were anesthetized with ether and perfused through the heart with isotonic saline followed by $10 \% \mathrm{formol} / \mathrm{saline}$. The $\mathrm{L}_{5}$ DRG pair which provide the main fiber contribution to the sciatic nerves and the SCG were dissected out to be analyzed for size and morphology. The contralateral DRG served as a control for the axotomized DRG. The ganglia were placed in fixative for 1 or 2 days, then dehydrated through graded ethanol, and embedded in paraffin. Serial $8-\mu \mathrm{m}$ sections were cut and stained with $0.1 \%$ toluidine blue.

To ascertain the total number of sensory neurons in the $L_{5} D R G$, neurons with discernible nucleoli were counted on every 10th section at a magnification of $\times 200$. Despite the heterogeneity of cell size in the DRG, identification of normal DRG neurons was straightforward. All of the neurons contained prominent Nissl substance and a large nucleus with one or two nucleoli. Glia cells and other non-neuronal cells were clearly distinguishable and were omitted from counting. The correction factor for split nucleoli was calculated according to the method of Konigsmark (1970). For neuronal perikaryal size measurements, the cell profiles were drawn with a camera lucida using a $\times 20$ objective. The area of each drawing was determined by means of a computerized graphic analyzer (Summagraphics Corp., Fairfield, CT). In each ganglion, 200 to 400 neurons were measured. The measurements were made on all cells in one or two separate histological sections from each ganglion to ensure that neurons from all over the ganglion were sampled.

In all morphological measurements, the slides were coded until the experiment was concluded. Statistically significant differences were determined by the Student's $t$ test (two-tailed).

\section{Results}

Contribution of $L_{5} D R G$ neurons to the sciatic nerve. After 24 $\mathrm{hr}$ the localized injections of HRP into the crushed sciatic nerves labeled most of the neurons in $\mathrm{L}_{4}$ and $\mathrm{L}_{5} \mathrm{DRG}$. In the $\mathrm{L}_{5}$ DRG, $82 \%$ of the neurons (527 of 641 neurons counted in three animals) were labeled. Granular reaction product could be detected in both small and large neurons; however, tracer accumulation was more conspicuous in the small neurons. No HRP granules were seen in the contralateral ganglia. The results in this experiment confirmed the anatomical assumption that the majority of the neurons $(>80 \%)$ in $L_{5}$ DRG projected their axons into the sciatic nerve.

Effects of NGF antiserum on survival of postnatal DRG neurons. Newborn rats received daily injections of NGF antiserum $(150 \mu \mathrm{l})$ for 7 days and were sacrificed on PND-13. Controls received an equal volume of nonimmunized serum. Treatment with NGF antiserum caused a marked atrophy of the SCG when compared under the dissecting microscope with the control serum-treated animals, whereas the size reduction of the DRG between these two groups of pups was much less pronounced. These gross changes were supported by the histological data. Longitudinal sections of SCG revealed a reduction in both number and size of the sympathetic neurons. The total number of neurons in the SCG was decreased by $93 \%$ (Fig. 1). NGF antiserum also caused a significantly $(p<0.02)$ reduction in the total number of sensory neurons in the $L_{5}$ DRG by about $18 \%$ (Fig. 2). In all $\mathrm{L}_{5}$ DRG from the antiserum-treated animals, a consistent shift toward larger sizes, relative to the control serum-treated animals, was observed (Fig. 3). The relative numbers of medium and large neurons were increased and the proportion of small neurons was decreased. Thus, the decrease 


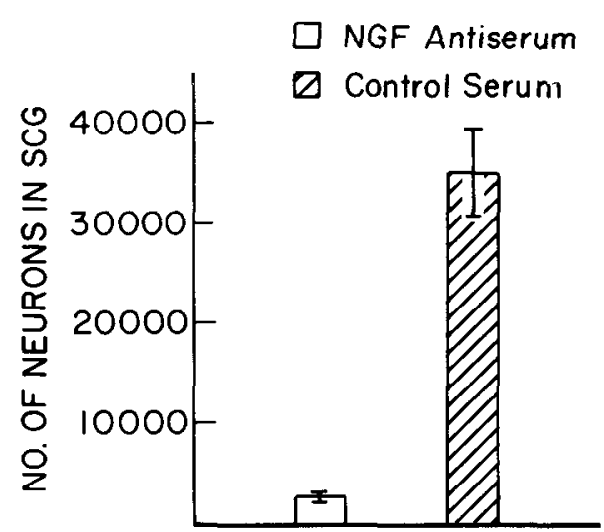

Figure 1. The effects of NGF antiserum on neuronal number in SCG of PND-13 rats. The number of neurons was determined as described under "Materials and Methods." Animals were treated with NGF antiserum (titer $>1000$ ) or normal, control serum. NGF antiserum ( $150 \mu \mathrm{l} /$ day) or control serum treatment was given on PND-0 through PND-6, and the animals were killed 1 week after the cessation of treatments. Values represent mean $\pm \mathrm{SEM}$ for groups of four to six ganglia.

\section{NGF Antiserum D. Control Serum}

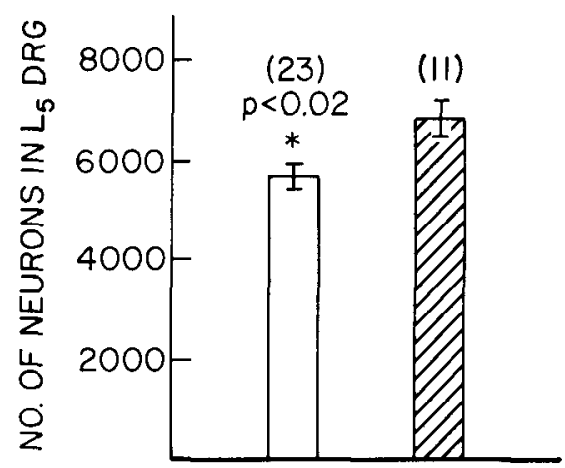

Figure 2 . The effects of NGF antiserum on neuronal number in normal, uncrushed $\mathrm{L}_{5}$ DRG of PND-13 rats. Animals were treated as described in the legend to Figure 1. Values represent mean \pm SEM; the number of animals in each group is shown in parentheses. The difference in neuronal number between the treated and untreated DRG is statistically significant $(*, p<0.02)$.

$(\mathbf{1 8 \%})$ in the number of neurons represents primarily a loss of small sensory neurons.

Time course of neuronal cell loss in $L_{5} D R G$ after axotomy. The right sciatic nerves of newborn rats were crushed on the day of birth (PND-0), and the animals were sacrificed at different time intervals after the crush $(0.5,1,3,4,6,8,10,12$, and 14 days). The $L_{5}$ DRG was dissected out and prepared for histological studies. The unoperated, contralateral DRG was used as control.

Crushing the sciatic nerve on the day of birth resulted in a profound loss of neurons. The number of neurons recognizable by conventional criteria decreased rapidly (Fig. 4). No neuronal death was observed in the normal DRG after birth. Forty-five percent of DRG neurons were lost 1 day after the sciatic nerve crush. After the first day there was no further neuronal loss, since the total number of neurons in the axotomized DRG remained at a constant level of about 40 to $50 \%$ of the controls for the whole 2-week interval studied (Fig. 4). The axotomized DRG neurons in the neonatal animals did not show the typical basophilia seen in axotomized adult neurons. The nucleoli appeared smaller when compared with the normal control.

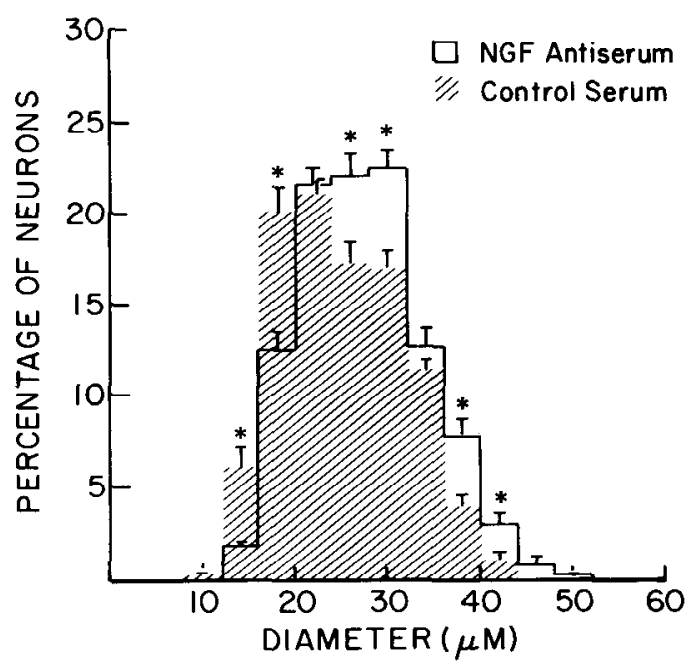

Figure 3. Histograms illustrating the size spectrum of neuronal perikarya in $L_{5}$ DRG of NGF antiserum-treated and control animals 1 week after the treatment (i.e., PND-13). Animals were treated as described in the legend to Figure 1. Values represent mean \pm SEM for groups of ganglia indicated in Figure 1. Significant difference $(p<0.05$ to 0.001 ) in neuronal number between the experimental and control ganglia is denoted by asterisks.

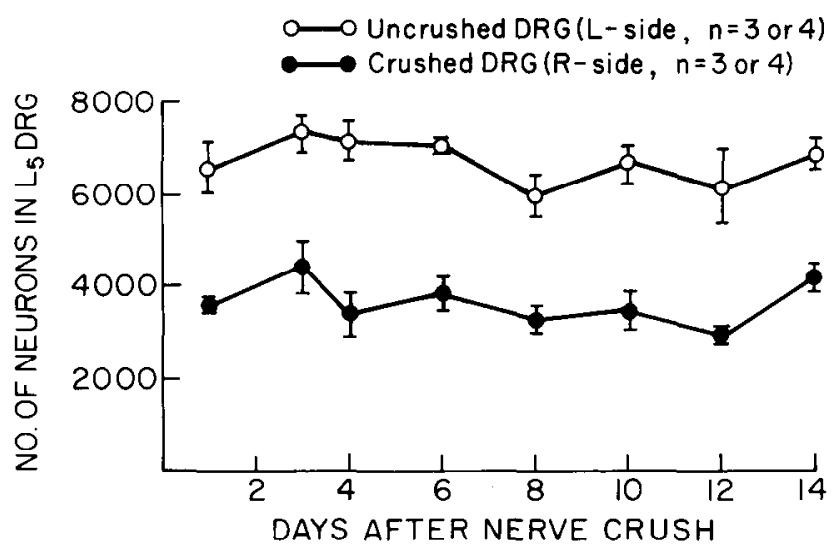

Figure 4. Time course of neuronal loss in $\mathrm{L}_{5} \mathrm{DRG}$ after unilateral sciatic nerve crush in PND-0 rats. Animals were killed at various times as indicated. $L$-side, left, uncrushed side; $R$-side, right, crushed side. The difference between the crushed and uncrushed sides is statistically significant $(p<0.001)$ at all time points examined.

Effects of NGF antiserum on neuronal number in $L_{5} D R G$ after axotomy. Having shown that administration of NGF antiserum to newborn rats caused a significant decrease of sensory neurons in the DRG (Fig. 2), experiments were performed to determine whether NGF antiserum treatment would increase neuronal death in the axotomized DRG. Sciatic nerves of neonatal rats were unilaterally crushed and the animals were injected daily with $150 \mu \mathrm{l}$ of NGF antiserum for 7 days. The animals were killed 1 week after discontinuing the treatment.

Cell counts in $\mathrm{L}_{5}$ DRG from axotomized animals at PND-13 revealed no significant difference $(p>0.1)$ in the reduction of neuronal number between the antiserum-treated and the control serum-treated animals (Fig. 5). Both groups had a $40 \%$ reduction in neuronal number after nerve crush. Thus, exposure to NGF antiserum did not accentuate the cell loss caused by axotomy. However, as seen in the $L_{5}$ DRG of uninjured animals, axotomized ganglia from anti-NGF-treated animals showed the same decrease $(19 \%)$ compared with axotomized ganglia from control animals (Fig. 5). Thus, exposure to anti-NGF in either uninjured or axotomized ganglia results in a 15 to $20 \%$ decrease in cell numbers. 
NGF Antiserum

Control Serum

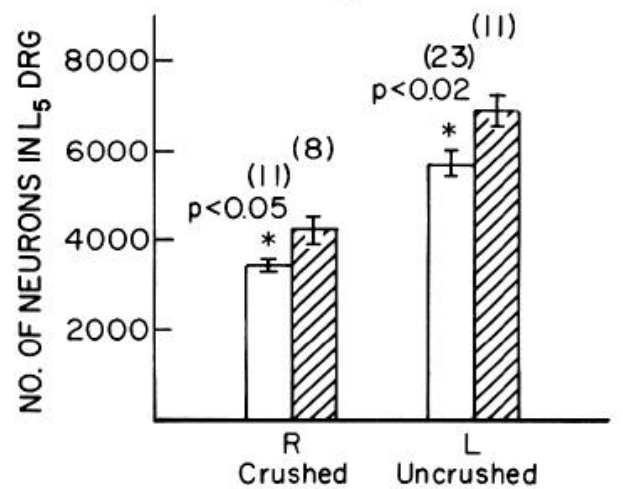

Figure 5. The effects of NGF antiserum on neuronal number in $\mathrm{L}_{5}$ DRG in PND-13 rats after right sciatic nerve crush as neonates. $R$, right, crushed side; $L$, left, uncrushed side. Animals were treated with NGF antiserum or control serum as described in the legend to Figure 1. Values represent mean $\pm \mathrm{SEM}$, and the number of animals for each group is indicated in parentheses. The difference in neuronal number between the NGF antiserum-treated and control serum-treated DRG is statistically significant $(*)$ for the axotomized DRG $(p<0.05)$ and for the unaxotomized DRG $(p<0.02)$.

Effects of NGF on neuronal number in $L_{5} D R G$ after axotomy. NGF has been shown to prevent cell death resulting from axotomy of the SCG (Hendry, 1975). It was of interest to investigate whether the administration of this specific growth factor would also protect the sensory neurons in the DRG from the destructive effect of axotomy despite the apparent lack of dependence of the majority of these cells on NGF for survival. This possibility was examined by studying the ability of NGF to prevent neuronal loss in the $\mathrm{L}_{5}$ DRG after sciatic nerve crush in neonatal rats. The animals were injected subcutaneously with either $100 \mu \mathrm{g}$ of NGF or vehicle (PBS) daily for 7 days. The animals were killed on PND-7.

Axotomy on PND-0 caused a marked atrophy of the $\mathrm{L}_{4}$ to $\mathrm{L}_{6}$ DRG when compared with the contralateral, unoperated controls under the dissecting microscope (Fig. $6 b$ ), as previously shown. Administration of NGF partially prevented the atrophy of the axotomized ganglia (Fig. $6 a$ ). The size of these ganglia, although slightly smaller than the contralateral controls, is definitely larger than the axotomized, vehicle-treated ganglia
(Fig. 6, $a$ and $b$ ). NGF also caused a hypertrophy of the SCG when compared with the controls.

Cell counts from PND-7 (Fig. 7) show that, as before (Fig. 4), $50 \%$ of neurons die in the absence of exogenous NGF. In contrast, only $20 \%$ of cells died in the animals treated with NGF, indicating that under these conditions NGF "saved" about two-thirds of the cells which would have died. Most of the surviving neurons remained basophilic and the neurons appeared to be hypertrophied. The axotomized, vehicle-treated DRG neurons were less intensely stained when compared to the normal controls, and the intercellular space was increased due to neuronal loss.

To determine whether the protective effects of NGF are of a permanent or temporary nature, a second group of axotomized animals was treated daily with NGF for 7 days and the animals were examined 3 weeks after the end of the treatment. Upon gross inspection, the size of the axotomized ganglia of this group was similar to that of the axotomized ganglia of the vehicle-treated group: both were greatly reduced in size. Cell counts in the NGF-treated ganglia showed a significant $(50 \%)$ decrease of neuronal number (Fig. 7), the same decrease as seen in vehicle-treated animals.

To further define the time course of neuronal death after axotomy and the ability of NGF to prevent that cell death, events occurring within the first $24 \mathrm{hr}$ after axotomy were examined. The pups were treated with NGF (100 $\mu \mathrm{g})$ or vehicle and the right sciatic nerves of newborn rats were axotomized. The animals were killed 12 or $24 \mathrm{hr}$ after the sciatic nerve crush. At the end of $12 \mathrm{hr}$, there was a significant (18\%) drop of neuronal number in the vehicle-treated, axotomized DRG (Table I), and by $24 \mathrm{hr}$ the number of cells was decreased by $35 \%$, a decrease similar to that seen in previous experiments (Fig. 4). In contrast, in axotomized animals treated with NGF, there was no decrease in neuronal number over the $24-\mathrm{hr}$ period (Table I), indicating that exogenous NGF, at least temporarily, completely prevents the cell death caused by axotomy of DRG neurons.

\section{Discussion}

The results of the present study support the view that NGF is essential not only for the survival of DRG sensory neurons during their embryonic development but also for the postnatal maturation and maintenance of some of these neurons. This is suggested by the observation of neuronal loss in the $\mathrm{L}_{5}$ DRG after the administration of NGF antiserum to the newborn

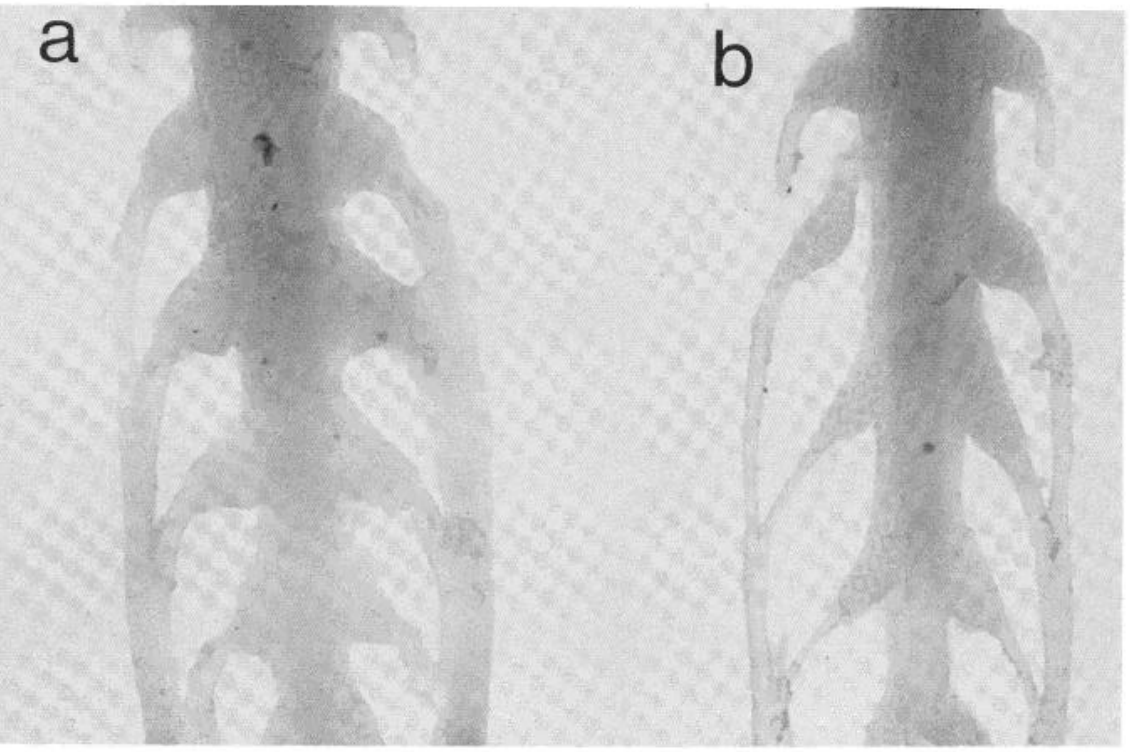

Figure 6. Whole amounts of PND-7 rat spinal cords with $\mathrm{L}_{3}$ to $\mathrm{L}_{6}$ ganglia attached (ventral view). The right sciatic nerves were crushed in PND-0 rats and treated either with $100 \mu \mathrm{g}$ of NGF or with vehicle for 7 days. The animals were killed on the 8 th day after the treatment. Note the gross size difference of $\mathrm{L}_{3}$ to $\mathrm{L}_{6}$ ganglia in NGF-treated, axotomized animals $(a)$ and in vehicle-treated, axotomized animals $(b)$. Magnification $\times 10$. 


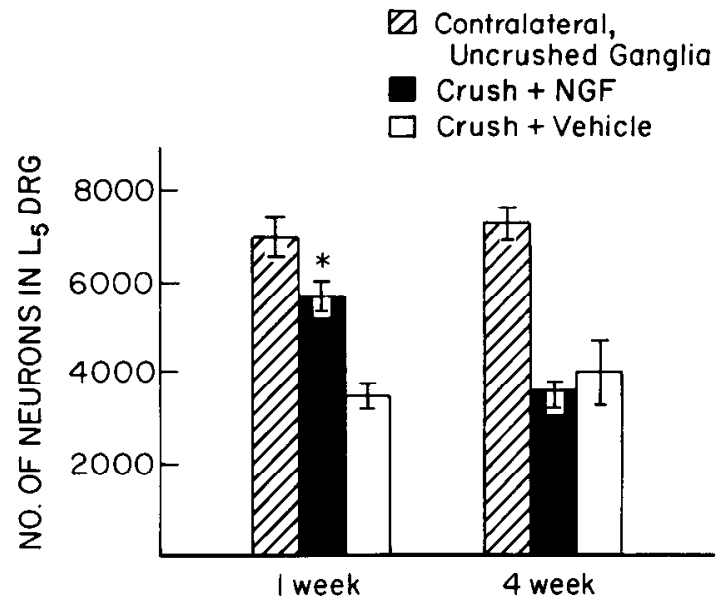

Figure 7. Effect of NGF treatment on the number of neurons in $\mathrm{L}_{5}$ DRG at 1 week and at 4 weeks after sciatic nerve crush. One group of animals was treated as described in the legend to Figure 6. A second group of animals was treated for 1 week and killed at 4 weeks of age. Values represent mean \pm SEM of groups of three to six ganglia.

TABIE I

Effect of axotomy and treatment with $N G F$ on the neuronal number in the $L_{5} D R G$ of neonatal rats

The right sciatc nerves were crushed in PND-0 rats. The animals were killed 12 or $24 \mathrm{hr}$ after the crush. For the 24 -hr time point, one group of animals was treated with $100 \mu \mathrm{g}$ of NGF before the surgery. Neuronal counts were determined as described under "Materials and Methods." The values given represent mean \pm SEM.

\begin{tabular}{|c|c|c|c|}
\hline \multirow{2}{*}{ Treatmeat } & \multirow{2}{*}{$\begin{array}{c}\text { Survival Time } \\
\text { after } \\
\text { Axotomy (hr) }\end{array}$} & \multicolumn{2}{|c|}{ Neuronal Number in $L_{5} D R G$} \\
\hline & & Left & Right $^{a}$ \\
\hline PBS & 12 & & $4730 \pm 315(6)^{b_{r} c}$ \\
\hline PBS & 24 & $5711 \pm 212(15)^{d}$ & $3765 \pm 104(6)^{c}$ \\
\hline NGF $(100 \mu \mathrm{g})$ & 24 & & $5423 \pm 145(5)$ \\
\hline
\end{tabular}

${ }^{*}$ Lesioned side.

${ }^{b}$ Numbers in parentheses, number of animals.

' Significantly different $(p<0.001)$ from controls.

${ }^{d}$ Average number of neurons in the unoperated, contralateral DRG.

rats. In contrast to previous statements (Levi-Montalcini and Angeletti, 1966) and findings (Johnson et al., 1980) in which no effect on postnatal DRG neurons could be observed after NGF antiserum administration, we found that the neuronal number in $\mathrm{L}_{5} \mathrm{DRG}$ was reduced by $18 \%$ after a 7 -day treatment with antiserum. This discrepancy may be because of the different quantities and titers of NGF antiserum used in the experiments. It is possible that a much higher titer of antibody is required to prevent the action of NGF on the DRG in the newborn animals. In the present experiment we have used sera with high titers of antibody ( $>4000)$ against mouse NGF. Thus, the administration of large quantities of high-titer NGF antiserum to neonatal rats not only produced massive destruction of sympathetic ganglia but also caused a loss of DRG neurons. The fact that the loss of sensory neurons was not large suggests that only a small population of sensory neurons is still dependent on NGF for survival during early postnatal development.

Morphometric analysis showed a shift toward a larger-sized neuronal population; at the same time, there was a decrease in the small cell population suggesting a loss of small neurons. The most probable explanation for this result is that the small sensory neurons are more susceptible to NGF deprivation in the postnatal period; this is consistent with the results obtained from experiments in which the animals were exposed to maternal anti-NGF antibodies (Johnson et al., 1980) that caused a neuronal loss greater among the small cell population.
The modest decrease in the number of $\mathrm{L}_{5}$ DRG neurons after NGF antiserum treatment demonstrates that the postnatal DRG neurons do require NGF for survival and that the distinction between prenatal and postnatal is arbitrary. Probably all DRG neurons go through a phase of NGF dependence. This dependent phase appears to be early in gestation for larger neurons (which are born earlier) and also probably shows a rostral-caudal gradient. Our detection of neuronal loss in the small cell population of a caudal ganglion is largely a reflection of when parturition occurs in a particular species relative to this continuous reduction in NGF dependence occurring in the large-small and rostral-caudal gradients.

Previous observations have demonstrated that axotomy or loss of peripheral target influence caused massive cell death in many developing nervous systems (Hamburger, 1934; Prestige, 1974). It has been postulated that an intact connection between the neuron and its peripheral target organ is required for the survival of the neuron during the early postnatal period. This contact may also be necessary for the final maturation of the DRG neurons. In the absence of this contact, these neurons will die or fail to develop normally. This is supported by our observation of a 40 to $50 \%$ cell loss in the $\mathrm{L}_{5} \mathrm{DRG}$ as a result of axotomy. Our results have shown a remarkably rapid degeneration of peripheral sensory neurons after a lesion of the sciatic nerve. About $20 \%$ of these neurons, whose cell bodies in the DRG reside quite a distance $(14 \mathrm{~mm})$ from the lesion site, die within $12 \mathrm{hr}$ of the axotomy. Essentially all of the DRG neurons which were to die (40 to $50 \%$ of the ganglion) degenerated within the first day after the nerve crush. The disappearance of the DRG neurons after axotomy is not unexpected; however, the rapidity of this destruction is surprising. Since the immature synthetic machinery in developing neurons is highly sensitive and is rapidly affected by changes in the peripheral target field (LaVelle, 1964), the immediate effects of axonal interruption may render them yet more vulnerable, initiating their rapid degeneration. The absence of characteristic chromatolytic patterns in the immature DRG neurons after axonal injury suggests a fundamental difference in cell metabolism for neurons still in an active growing and differentiating state (LaVelle and Sechrist, 1970). This difference may be largely due to an underdeveloped rough endoplasmic reticulum system in the developing neurons (Pilar and Landmesser, 1976). The time course of degeneration of an individual neuron may be so fast that it is difficult to "capture" a rapidly degenerating neuronal population at a specific time point when examined under the light microscope (Lieberman, 1971).

Since administration of NGF antiserum to the neonatal rats did not result in an enhancement of neuronal death after axotomy, our results do not support the hypothesis that neuronal injury causes neurons to "regress" back to a less mature state in which they require NGF for survival. However, following NGF antiserum administration, we found that the neuronal number in the axotomized DRG was reduced to the same level $(19 \%)$ as in the unaxotomized DRG $(18 \%)$; thus, axotomy does not appear to affect the dependence or lack of dependence of postnatal DRG neurons for NGF.

Previous studies have demonstrated that the destructive effect of axotomy on developing sympathetic neurons can be prevented by NGF treatment (Aloe et al., 1975; Hendry, 1975; Hendry and Campbell, 1976). To assess the role of NGF in counteracting the degenerative changes brought about by axotomy in the postnatal DRG, neonatal rats were treated with a high dose of exogenous NGF $(100 \mu \mathrm{g})$ for 7 consecutive days. Administration of NGF significantly reduced cell death in the DRG after axotomy, although NGF treatment alone did not change the neuronal number in the unaxotomized DRG. The results suggest that pharmacological amounts of NGF can markedly reduce the severity of the cell body response to 
axotomy. In fact, $24 \mathrm{hr}$ after axotomy, NGF treatment provided complete protection from neuronal death in DRG. This suggests that, although most rat DRG neurons no longer need NGF to survive after parturition, this neurotrophic factor can prevent, at least temporarily, cell death as a result of injuries to peripheral axons in the neonate. Cessation of the NGF treatment resulted in a marked reduction of DRG neurons to a number comparable to that of the untreated control when examined 3 weeks after the 7-day treatment. This temporary protective effect of NGF on the axotomized neurons may indicate that removal of NGF before the appropriate connections are established with the peripheral target organs will result in cell death. Previous studies have shown that postnatal development of DRG neurons in rats was completed in the first 15 days (Yamadori, 1970). In addition, it would appear that sensory innervation of the periphery by DRG neurons continues through the time of birth and takes about 2 weeks to acquire a mature pattern. If this is true, it may be possible to protect more of the NGF-responsive neurons from the destructive effects of axotomy by treating neonatal animals through this critical period. Experiments are presently underway to determine the effect of axotomy and NGF treatment on the number of surviving DRG neurons at different ages.

These studies demonstrate that the effects of exogenous NGF are not always the opposite of the effects produced by NGF antiserum. This is reflected by the finding that there is only a modest decrease (18\%) of DRG neurons in both unaxotomized and axotomized animals after NGF antiserum treatment. However, administration of NGF can, at least temporarily, completely prevent the death of the $50 \%$ of neurons in the DRG which would have died after axotomy. NGF antiserum causes deprivation of endogenous NGF in the animals and, therefore, defines the physiological role of NGF. The action of exogenous NGF is suggestive of the physiological role but demonstrates only a pharmacological effect of the agent.

In conclusion, our results demonstrate that axotomy of the sciatic nerve in newborn rats results in the destruction of lumbar DRG neurons. Administration of NGF antiserum decreases neuronal number in the normal DRG. However, NGFantiserum treatment does not increase the destructive effect of axotomy. Treatment with a large amount of exogenous NGF can temporarily antagonize the effect of axotomy. A sensitive period exists during which treatment with NGF is capable of maintaining at least some of the neurons that would otherwise degenerate as a result of the insult. It will be interesting to see whether administration of NGF for a longer period of time can permanently protect DRG neurons from the effects of axotomy.

\section{References}

Aloe, L., E. Mugnaini, and R. Levi-Montalcini (1975) Light and electron microscopic studies of the excess growth of sympathetic ganglia in rats injected daily from birth with 6-OHDA and NGF. Arch. Ital. Biol. 113: 236-253.

Aloe, L., C. Cozzari, P. Calissano, and R. Levi-Montalcini (1981) Somatic and behavioral postnatal effects of fetal injections of nerve growth factor antibodies in the rat. Nature 291: 413-415.

Bocchini, F., and P. U. Angeletti (1969) The nerve growth factor: Purification as a 30,000 molecular weight protein. Proc. Natl. Acad. Sci. U. S. A. 64: 787-794.

Bodian, D. (1947) Nucleic acid in nerve regeneration. Soc. Exp. Biol. Symp. 1: 163-178.

Brattgard, S. O., J. E. Edström, and H. Hydén (1957) The chemical changes in regenerating neurons. J. Neurochem. 1: 316-325.

Carmel, P. W., and B. M. Stein (1969) Cell changes in sensory ganglia following proximal and distal nerve section in the monkey. J. Comp. Neurol. 135: 145-166.

Cragg, B. G. (1970) What is the signal for chromatolysis? Brain Res. 23: 121.

Ennis, M., F. L. Pearce, and C. A. Vernon (1979) Some studies on the mechanism of action of antibodies to nerve growth factor. Neuroscience 4: 1391-1398.

Fenton, E. L. (1970) Tissue culture assay of nerve growth factor and of the specific antiserum. Exp. Cell Res. 59: 383-392.

Goedert, M., U. Otten, T. H. Schäfer, M., Schwab, and H. Thoenen (1980) Immunosympathectomy: Lack of evidence for a complementmediated cytotoxic mechanism. Brain Res. 201: 399-409.

Goedert, M., K. Stöckel, and U. Otten (1981) Biological importance of the retrograde axonal transport of nerve growth factor in sensory neurons. Proc. Natl. Acad. Sci. U. S. A. 78: 5895-5898.

Gorin, P. D., and E. M. Johnson, Jr. (1979) Experimental autoimmune model of nerve growth factor deprivation: Effects on developing peripheral sympathetic and sensory neurons. Proc. Natl. Acad. Sci. U. S. A. $76: 5382-5386$.

Gorin, P. D., and E. M. Johnson, Jr. (1980) Effects of long-term nerve growth factor deprivation on the nervous system of the adult rat: An experimental autoimmune approach. Brain Res. 198: 27-42.

Hamburger, V. (1934) The effects of wing bud extirpation on the development of the central nervous system in chick embryos. J. Exp. Zool. 68: 449-473.

Hamburger, V., and R. Levi-Montalcini (1949) Proliferation, differentiation and degeneration in the spinal ganglia of the chick embryo under normal and experimental conditions. J. Exp. Zool. 111: 457501.

Hamburger, V., J. K. Brunso-Bechtold, and J. W. Yip (1981) Neuronal death in the spinal ganglia of the chick embryo and its reduction of nerve growth factor. J. Neurosci. 1: 60-70.

Hare, W. K., and J. C. Hinsey (1940) Reactions of dorsal root ganglion cells to section of peripheral and centrl processes. J. Comp. Neurol. 7: 489-502.

Hendry, I. A. (1975) The response of adrenergic neurons to axotomy and nerve growth factor. Brain Res. 94: 87-97.

Hendry, I. A., and J. Campbell (1976) Morphometric analysis of rat superior ganglion after axotomy and nerve growth factor treatment. J. Neurocytol. 5: 351-360.

Hendry, I. A., K. Stöckel, H. Thoenen, and L. L. Iversen (1974) The retrograde axonal transport of nerve growth factor. Brain Res. 68: 103-121.

Hollyday, M., and V. Hamburger (1976) Reduction of naturally occurring motor neuron loss by enlargement of the periphery. J. Comp. Neurol. 170: 311-320.

Houthoff, H. J., and J. Drukker (1977) Changing patterns of axonal reaction during neuronal development. A study in the developing chick nervous system. Neuropathol. Appl. Neurobiol. 3: 441-452.

Johnson, E. M. (1978) Destruction of the sympathetic nervous system in neonatal rats and hamsters by vinblastine: Prevention by concomitant administration of nerve growth factor. Brain Res. 141: 105118.

Johnson, E. M., R.Y. Andres, and R. A. Bradshaw (1978) Characterization of the retrograde transport of nerve growth factor (NGF) using high specific $\left[{ }^{125} \mathrm{I}\right]$ NGF. Brain Res. 150: $319-331$.

Johnson, E. M., P. D. Gorin, L. D. Brandeis, and J. Pearson (1980) Dorsal root ganglion neurons are destroyed by exposure in utero to maternal antibody to nerve growth factor. Science 210:916-918.

Kessler, J. A., and I. B. Black (1980) Nerve growth factor stimulates the development of substance $\mathrm{P}$ in sensory ganglia. Proc. Natl. Acad. Sci. U. S. A. 77: 649-652.

Konigsmark, B. W. (1970) Methods for the counting of neurons. In Contemporary Research Methods in Neuroanatomy, W. J. H. Nauta and S. O. E. Ebbsson, eds., pp. 315-310, Springer-Verlag, New York. Kornblum, H. I., and E. M. Johnson, Jr. (1982) Time and dose dependence of effects of nerve growth factor on sympathetic and sensory neurons in neonatal rats. Brain Res. 234: 41-51.

Landmesser, L., and G. Pilar (1978) Interactions between neurons and their targets during in vivo synaptogenesis. Fed. Proc. 37: 2016-2022.

LaVelle, A. (1964) Critical periods of neuronal maturation. Prog. Brain Res. 9: $93-96$.

LaVelle, A., and J. W. Sechrist (1970) Immature and mature reaction patterns in neurons after axon section. Anat. Rec. 166: 355 .

Levi-Montalcini, R., and P. U. Angeletti (1966) Immunosympathectomy. Pharmacol. Rev. 18: 619-628.

Lieberman, A. R. (1971) The axon reaction. A review of the principal features of perikaryal responses to axon injury. Int. Rev. Neurobiol. 14: 49-124.

Lieberman, A. R. (1974) Some factors affecting retrograde neuronal 
responses to axonal lesions. In Essay on the Nervous System, R. Bellairs and E. G. Gray, eds., pp. 71-105, Clarendon Press, Oxford.

Mesulam, M. -M. (1978) Tetramethylbenzidine for horseradish peroxidase neurochemistry. A non-carcinogenic blue reaction product with superior sensitivity for visualizing neural afferents and efferents. J. Histochem. Cytochem. 26: 106-117.

Murray, M., and B. Grafstein (1969) Changes in the morphology and amino acid incorporation of regenerating goldfish optic neurons. Exp. Neurol. 23: 544-560.

Otten, U., M. Goedert, N. Mayer, and F. Lembeck (1980) Requirement of nerve growth factor for development of substance P-containing sensory neurons. Nature 287: 158-189.

Otten, U., H. P. Lorez, and F. Businger (1983) Nerve growth factor antagonizes the neurotoxic action of capsaicin on primary sensory neurones. Nature $301: 515-517$

Pannese, E. (1963) Investigation on the ultrastructural changes of the spinal ganglion neurons in the course of axon regeneration and cell hypertrophy. I. Changes during axon regeneration. Z. Zellforsch. Mikrosk. Anat. 60: 711-740.

Paravicini, U., K. G. Stöckel, and H. Thoenen (1975) Biological importance of retrograde axonal transport of nerve growth factor in adrenergic neurons. Brain Res. 84: 279-291.

Pilar, G., and L. Landmesser (1976) Ultrastructural difference during embryonic cell death in normal and peripherally deprived ciliary ganglion. J. Cell Biol. 68: 339-356.
Prestige, M. C. (1974) Axon and cell numbers in the developing nervous system. Br. Med. Bull. 30: 107-111.

Ramón y Cajal, S. (1928) Degeneration and Regeneration of the Nervous System, R. M. May, ed. and transl., Vol. 1, Oxford University Press, London.

Schwartz, J. P., J. Pearson, and E. M. Johnson (1982) Effect of exposure to anti-NGF on sensory neurons of adult rats and guinea pigs. Brain Res. 244: 378-381

Stöckel, K., M. E. Schwab, and H. Thoenen (1975) Specificity of retrograde transport of nerve growth factor (NGF) in sensory neurons: A biochemical and morphological study. Brain Res. 89: 1-14.

Thoenen, H., and Y. -A. Barde (1980) Physiology of nerve growth factor. Physiol. Rev. 60: 1284-1335.

Thoenen, H., U. Otten, and M. E. Schwab (1979) Orthograde and retrograde signals for the regulation of neuronal gene expression: The peripheral sympathetic nervous system as a model. in The Neurosciences: Fourth Study Program, F. O. Schmitt and F. G. Worden, eds., pp. 911-928, MIT Press, Cambridge, MA.

Watson, W. E. (1965) An autoradiographic study of the incorporation of nucleic acid precursors by neurons and glia during nerve regeneration. J. Physiol. (Lond.) 180: 741-754.

Watson, W. E. (1974) Cellular responses to axotomy and related procedures. Br. Med. Bull. 30: 112-115.

Yamadori, T. (1970) A light and electron microscopic study on the postnatal development of spinal ganglia in rats. Acta Anat. Nippon 45: 191-192. 